\title{
ON THE COHOMOLOGY OF FUCHSIAN GROUPS
}

\author{
by S. J. PATTERSON
}

(Received 12 September, 1974; revised 15 May, 1975)

1. Introduction. The object of this paper is to redevelop the classical theory of multipliers of Fuchsian groups [16] and to attempt a classification. The language which appears most appropriate is that of group extensions and the cohomology of groups. This viewpoint is not entirely novel [12] but the entire theory has never been based on it before.

A Fuchsian group $G$ is a discrete subgroup of $P S L(2 ; \mathbf{R})$. We shall further assume that $G$ is finitely generated and that $\operatorname{PSL}(2 ; \mathbf{R}) / G$ has finite volume. We shall begin by investigating the extensions of $P S L(2 ; \mathbf{R})$. These induce extensions of $G$.

On the other hand $G$ can be given explicitly by generators and relations. So, in principle at any rate, its extensions can be classified from the point of view of group theory. There is then the basic problem of relating these two approaches.

In the case that $G$ has no elliptic elements, this identification can be carried out explicitly by using Chern characters to identify algebraically and geometrically defined objects. A partial extension to groups with elliptic elements can be made using the transfer homomorphism of group cohomology.

As a first application, we consider the question as to when $G$ can be lifted to $S L(2 ; \mathbf{R})$. We prove again a theorem of Petersson ([17]) and Bers ([1]) (itself the answer to a question of Siegel ([22])). Bers' proof was an application of the theory of moduli of Riemann surfaces; ours, being intrinsic, can be considered rather more satisfying. Further, our results are more complete than Bers'. Petersson's proof is similar to, but rather more complicated than, ours.

The second application is a sketch of the theory of weights and multipliers for automorphic forms. This will be treated in two ways; one is the classical approach due to Petersson while the other is more in keeping with our general philosophy. This also has the virtue that it shows how the representation theory approach to the Selberg trace formula (see [6, Ch. 1]) ties up with Selberg's own approach in the case of arbitrary weights.

One of the most famous multiplier systems is that arising from the Dedekind $\eta$-function on the modular group. The appearance of the $\eta$-function in the Kronecker limit formula suggests, in view of Selberg's theory of Eisenstein series [20], that a similiar function might be defined on an arbitrary Fuchsian group. That this is so is shown by Goldstein [8] in terms of Eisenstein series. We shall give a new and much more general proof which uses the resolvent of the Laplace-Beltrami operator in place of Eisenstein series. This produces multiplier systems even when $G$ has no parabolic elements. We conclude by showing that our results include the Kronecker limit formula.

Notation. $G$ will denote a finitely generated Fuchsian group of the first kind. We shall write $\mathfrak{G}=\operatorname{PSL}(2 ; \mathbf{R})$ and $C_{n}$ for the cyclic group with $n$ elements. H will denote the upper half-plane on which $\mathfrak{G}$ will act in the usual way. 
2. Extensions of PSL $(2 ; \mathbf{R}) . \quad(\mathfrak{G}=P S L(2 ; \mathbf{R})$ acts on the upper half-plane. Let $M$ be the subgroup of elements of the form $m(a, b)(a, b \in \mathbf{R}, a>0)$, where

$$
m(a, b)(z)=a z+b .
$$

Let $K$ be the subgroup of 6 fixing $i$. Then the product map

$$
M \times K \rightarrow \mathbb{6}
$$

is well known to be a homeomorphism ("Iwasawa decomposition "). Furthermore $K \cong \mathbf{R} / \mathbf{Z}$. Let us write $S=\mathbf{R} / \mathbf{Z}$ which we call, as usual, the circle group. Note also that $(\mathfrak{S}$ has a trivial centre.

$M$ is homeomorphic to $\mathbf{R} \times] 0, \infty$ [ and is therefore contractible. Hence

$$
\pi_{1}(\mathfrak{G}) \cong \mathbf{Z} \text {. }
$$

Recall that an arbitrary covering manifold of a connected Lie group has a unique Lie group structure extending the given one. If we let $\boldsymbol{\sigma}_{\infty}$ be the universal covering space of $\boldsymbol{\sigma}$ and let, for $n>1, \mathfrak{G}_{n}$ be the unique covering space of degree $n$ of $\mathfrak{G}_{\text {. Then }} \boldsymbol{\sigma}_{\infty}$ and $\mathfrak{W}_{n}$ are covering groups of $\boldsymbol{6}$. We have exact sequences

$$
\begin{aligned}
& e \rightarrow Z \rightarrow \mathfrak{G}_{\infty} \rightarrow \mathfrak{W} \rightarrow e \\
& e \rightarrow C_{n} \rightarrow \mathfrak{G}_{n} \rightarrow \mathfrak{W} \rightarrow e .
\end{aligned}
$$

Let $H^{*}$ be the group cohomology functor. Then (2) and (3) give rise to elements of $H^{2}(\mathfrak{G}, \mathbf{Z})$ and $H^{2}\left(\mathfrak{G}, C_{n}\right)$ respectively. We would like to identify 2 -cocycles representing these.

Observe that, as $\mathscr{G}_{\infty}$ and $\mathfrak{G}_{n}$ are connected, they act trivially on $\mathbf{Z}$ and $C_{n}$. In particular, $\mathbf{Z}$ (resp. $C_{n}$ ) is the centre of $\mathfrak{G}_{\infty}$ (resp. $\left.\mathfrak{G}_{n}\right)$.

We shall now define a 2 -cocycle on $\mathfrak{G}$ and verify that this defines $\mathfrak{G}_{\infty}$. $\quad \mathfrak{G}$ acts on $\mathbf{H}$. Define $\log$ as that branch taking its values in $\{z: 0 \leqq \operatorname{Im}(z)<2 \pi\}$. Choose $w \in \mathbf{H}$ and define, for $g, h \in \mathbb{0}$,

$$
2 \pi i \cdot a(g, h)=\log (g h)^{\prime}(w)-\log g^{\prime}(h(w))-\log h^{\prime}(w) .
$$

By the chain rule, this is an integer and it is easy to see that it does not depend on the choice of $w$. It is also immediate that $a$ is a 2-cocycle.

Let $\mathfrak{G}(a)$ be the extension

$$
e \rightarrow \mathrm{Z} \rightarrow \mathfrak{W}(a) \rightarrow \mathfrak{W} \rightarrow e
$$

determined by $a$. It is easy to verify from $(4)$ that $(5(a)$ has a natural structure of a Lie group. $a$ restricted to $K$ or $M$ is again a 2-cocycle. So there are extensions

$$
\begin{aligned}
& e \rightarrow \mathbf{Z} \rightarrow M(a) \rightarrow M \rightarrow e \\
& e \rightarrow \mathbf{Z} \rightarrow K(a) \rightarrow K \rightarrow e,
\end{aligned}
$$

where, furthermore, $M(a)$ and $K(a)$ can be considered as subgroups of $(5(a)$. But $M$ is 
contractible and hence cohomologically trivial. Thus $M(a) \cong M \times \mathbf{Z}$. So $M$ can be lifted to a subgroup of $\mathfrak{G}(a)$. From (1) one deduces that the resulting product map

$$
M \times K(a) \rightarrow \mathfrak{G}(a)
$$

is a bijection.

We must now look at $K(a)$. There is a homomorphism $\theta$ from $\mathbf{R}$ into $K$ with kernel $\mathbf{Z}$ given by

$$
\theta(t)=(w \mapsto(w \cdot \cos \pi t+\sin \pi t) /(-w \cdot \sin \pi t+\cos \pi t)) .
$$

From (4) one sees that, on taking $w=i$,

$$
a(\theta(s), \theta(t))=(s+t)-(s)-(t)
$$

where, if $x \in \mathbf{R},(x) \equiv x(\bmod , \mathbf{Z})$ and $(x) \in[0,1[$. But this is the 2-cocycle on $S$ defining the extension

$$
e \rightarrow \mathbf{Z} \rightarrow \mathbf{R} \rightarrow S \rightarrow e
$$

and so $K(a) \cong \mathbf{R}$. By $(5), \mathfrak{E}(a)$ is simply connected and so is isomorphic to $\mathfrak{G}_{\infty}$.

Thus the 2-cocycle a given by (4) determines the extension (2).

We can also consider $a$ as an element of $\mathbf{Z} / n \mathbf{Z}$. Likewise we can show that as such it determines the extension (3).

Finally let us note that

$$
\mathfrak{G}_{2}=S L(2 ; \mathbf{R}) \text {. }
$$

3. Cohomology of Fuchsian groups.. A Fuchsian group $G$ can be generated by a system of generators of the form

$$
A_{1}, \ldots, A_{\theta}, \quad B_{1}, \ldots, B_{g}, \quad P_{1}, \ldots, P_{s}, \quad E_{1}, \ldots, E_{t}
$$

subject to the relations

$$
\left.\begin{array}{c}
{\left[A_{1}, B_{1}\right] \ldots\left[A_{g}, B_{g}\right] \cdot P_{1} \ldots P_{s} \cdot E_{1} \ldots E_{t}=I} \\
E_{j}^{e(j)}=I
\end{array}\right\}
$$

where $e(j)>1(1 \leqq j \leqq t)$ is a positive integer. The $P_{j}(1 \leqq j \leqq s)$ are parabolic and any parabolic element of $G$ is conjugate to some power of one of the $P_{j}$. Likewise the $E_{k}$ are elliptic and any elliptic element is conjugate to some power of one of the $E_{k}$. There may, of course, be no elliptic or parabolic elements, in which case we set $t=0$ or $s=0$. See, for example, $[16, \S 2.3]$.

If $D$ is a fundamental domain for $G$ on $\mathbf{H}$ and $\sigma$ is the hyperbolic area on $\mathbf{H}$ we have

$$
\sigma(D)=2 \pi\left((2 g-2)+s+\sum_{j=1}^{t}\left(1-e(j)^{-1}\right)\right) .
$$

In view of this let us write

$$
\tau(G)=2 g-2+s+\sum_{j=1}^{t}\left(1-e(j)^{-1}\right)
$$


This is a rational number which will play an important role. In particular, if $H$ is a subgroup of finite index in $G$, it is again a Fuchsian group and, by (10),

$$
\tau(H)=[G: H] \cdot \tau(G) .
$$

It is easy to calculate the second cohomology groups of $G$ given the presentation (8), (9). The method is given in [10, p. 131]. Let $M$ be an abelian group on which $G$ acts trivially. If we carry out the prescription in [10] we find:

Proposition 1. If $s>0$, then

$$
H^{2}(G, M) \cong \bigoplus_{j=1}^{\dagger} M / e(j) M .
$$

If $s=0, H^{2}(G, M)$ is the cokernel of the map $M^{t} \rightarrow M^{t+1}$ given by

$$
\left(m_{1}, \ldots, m_{\imath}\right) \mapsto\left(m_{1}+\ldots+m_{t}, e(1) m_{1}, \ldots, e(t) m_{t}\right) .
$$

Let us consider the special case $M=\mathbf{Z}$. If $s>0$ then $H^{2}(G, \mathbf{Z})$ is the finite torsion group $\underset{1 \leqq j \leqq t}{\oplus} C_{e(j)}$.

Now let $T(G)$ be the finite abelian group generated by $y_{1}, \ldots, y_{t}$ subject to the relations

$$
y_{j}^{e(j)}=I \quad(1 \leqq j \leqq t) .
$$

COROLLARY: If $s>0$, then

$$
H^{2}(G, \mathbf{Z}) \cong T(G)
$$

If $s=0$, then

$$
H^{2}(G, \mathbf{Z}) \cong \mathbf{Z} \oplus T(G) .
$$

(The appearance of a factor of $\mathbf{Z}$ when $s=0$ corresponds to the fact that $G \backslash \mathbf{H}$ is then compact.)

Proof. If $s \neq 0$ the result is already proved. So we may suppose that $s=0$. Let $\varphi: \mathbf{Z}^{t} \rightarrow \mathbf{Z}^{t+1}$ be the map defined in Proposition 1; then $H^{2}(G, \mathbf{Z})=\operatorname{Coker}(\varphi)$. Let

$$
F: \mathbf{Z}^{t+1} \rightarrow \mathbf{Z}^{t+1} ;\left(m_{0}, m_{1}, \ldots, m_{t}\right) \mapsto\left(m_{0}, e(1) m_{1}, \ldots, e(t) m_{t}\right) .
$$

Then

$$
\varphi\left(\mathbf{Z}^{t}\right) \subseteq F\left(\mathbf{Z}^{t+1}\right) \subseteq \mathbf{Z}^{t+1} .
$$

Hence

$$
F\left(\mathbf{Z}^{t+1}\right) / \varphi\left(\mathbf{Z}^{t}\right) \cong \mathbf{Z} \quad \text { and } \quad \mathbf{Z}^{t+1} / F\left(\mathbf{Z}^{t+1}\right) \cong T(G) .
$$

The conclusion follows by the general structure theory of abelian groups.

$G$ is a discrete subgroup of 5 . So we have a map

$$
\operatorname{Res}{ }_{G}^{\mathfrak{G}}: H^{2}(\mathfrak{G}, \mathbf{Z}) \rightarrow H^{2}(G, \mathbf{Z}) \text {. }
$$

We have computed the structure of $H^{2}(G, Z)$ and, in Section 2, we found a special element of $H^{2}(\mathfrak{G}, \mathrm{Z})$. Our immediate problem is to find where the image of this element $\operatorname{sits}$ in $H^{2}(G, \mathrm{Z})$. 
Results similar to those of this section have been found by Godement [7]. His methods are applicable to a very wide class of discrete groups but the conclusions are not quite so precise. However, he does calculate the higher cohomology groups of Fuchsian groups.

4. Surface groups. A Fuchsian group will be called a surface group if it has no elliptic or parabolic elements. In terms of the presentation (8), (9) this becomes $s=t=0$. By the corollary to Proposition $1, H^{2}(G, Z)=Z$. Let $\alpha$ be the element of $H^{2}(\mathfrak{G}, \mathbf{Z})$ corresponding to the 2-cocycle $a$ defined in (4).

Proposition 2. Let $G$ be a surface group. Then there is a generator $u$ of $H^{2}(G, \mathbf{Z})$ so that

$$
\operatorname{Res}{ }_{G}^{\sigma}(\alpha)=(2 g-2) \cdot u .
$$

Proof. Let $X=G \mid \mathbf{H}$ be the Riemann surface corresponding to $G$. Let $\pi: \mathbf{H} \rightarrow X$ be the projection map. Let $\Omega^{1,0}(X)$ be the line bundle of forms of type $(1,0)$. We shall identify $H^{2}(X, \mathbf{Z})$ and $H^{2}(G, \mathbf{Z})$ and calculate the Chern class of $\Omega^{1,0}(X)$ in two different ways. See [2, p. 33].

Let $D$ be a fundamental domain for $G$. If, for $z \in \mathbf{H}$, we write $z=x+i y$, then

$$
d \sigma=y^{-2} d x \wedge d y
$$

is the area associated to the Poincaré metric. Let $f_{1}, f_{2}$ be two sections of $\Omega^{1,0}(X)$ lifted to $\mathbf{H}$ in the usual way. Then

$$
\left(f_{1}, f_{2}\right)=\int_{D} f_{1}(z) \cdot f_{2}(z) y^{2} d \sigma(z)
$$

is invariantly defined and defines a Hermitian structure on $\Omega^{1,0}(X)$. By [2, p. 45] the curvature form of this Hermitian structure is

But if $F$ is any function, then

$$
K=(-1 / 2 \pi i) \partial \tilde{\partial} \log y^{2} .
$$

Thus

$$
\partial \bar{\partial} F=-(i / 2)\left(F_{x x}+F_{y y}\right) d x \wedge d y .
$$

$$
K=(1 / 2 \pi)(-d \sigma)
$$

$K$ is the representative of the Chern class of $\Omega^{1,0}(X)$ in the de Rham cohomology group $H^{2}(X, \mathbf{R})$ (see [2, p. 49]). But $X$ is a two-dimensional manifold. So $H^{2}(X, \mathbf{Z}) \cong \mathbf{Z}$ and, if $[X]$ is the generator of $H_{2}(X, Z)$, then $H^{2}(X, Z)$ is generated by an element $\mu$, so that $\mu([X])=1$. Thus $K=N \cdot \mu$, where

$$
\begin{aligned}
N & =\int_{D} K \\
& =-\sigma(D) / 2 \pi \\
& =-(2 g-2) .
\end{aligned}
$$

Thus the Chern class of $\Omega^{1,0}(X)$ is $-(2 g-2) \mu$. 
Let $F$ be a sheaf on $X$ and let $\pi^{*} F$ be the pull-back to $\mathbf{H}$. Let $\Gamma\left(\mathbf{H}, \pi^{*} F\right)$ be the $G$-module of sections of $\pi^{*} F$. This is discussed in detail in [14, pp. 22-24] and we shall assume this discussion. The conditions there are satisfied and consequently

$$
H^{*}\left(G, \Gamma\left(\mathbf{H}, \pi^{*} F\right)\right) \rightarrow H^{*}(X, F)
$$

is an isomorphism of cohomology functors.

We shall consider the three sheaves $\mathbf{Z}, A(X)$, and $A^{\times}(X)$. $\mathbf{Z}$ is the constant sheaf, $A(X)$ is the sheaf of germs of $C^{\infty}$ functions on $X$ and $A^{\times}(X)$ is the sheaf of germs of invertible $C^{\infty}$ functions. Then $\Gamma\left(\mathbf{H}, \pi^{*} \mathbf{Z}\right) \cong \mathbf{Z}, \Gamma\left(\mathbf{H}, \pi^{*} A(X)\right) \cong C^{\infty}(\mathbf{H})$ and $\Gamma\left(\mathbf{H}, \pi^{*} A^{\times}(X)\right) \cong C^{\infty}(H)^{\times}$(the group of units of the ring $C^{\infty}(\mathbf{H})$ ). The $G$-action on $C^{\infty}(\mathbf{H})$ or $C^{\infty}(\mathbf{H})^{\times}$is given by

$$
(g \cdot f)(z)=f\left(g^{-1}(z)\right)
$$

A line bundle on $X$ is defined by an element of $H^{1}\left(X, A^{\times}(X)\right)$ (see [2, p. 31]). The short exact sequence of sheaves

$$
0 \rightarrow \mathbf{Z} \rightarrow A(X) \stackrel{e}{\rightarrow} A^{\mathrm{x}}(X) \rightarrow 0
$$

induces, via the cohomology exact sequence (see [2, p. 33]), an isomorphism

$$
\delta: H^{1}\left(X, A^{\times}(X)\right) \rightarrow H^{2}(X, \mathbf{Z}) \text {. }
$$

The element of $H^{2}(X, \mathbf{Z})$ corresponding to a line bundle is its Chern class.

Now, using the isomorphism (15), let us mimic this construction in $G$-modules. We have

$$
\begin{aligned}
& H^{2}(G, \mathbf{Z}) \cong H^{2}(X, \mathbf{Z}) \\
& H^{1}\left(G, C^{\infty}(\mathbf{H})\right) \cong H^{1}\left(X, A^{\times}(X)\right)
\end{aligned}
$$

Corresponding to (17), we have

$$
0 \rightarrow \mathbf{Z} \rightarrow C^{\infty}(\mathbf{H}) \stackrel{e}{\rightarrow} C^{\infty}(\mathbf{H})^{\times} \rightarrow 0,
$$

where $e(f)(z)=\exp (2 \pi i f(z))$. Again we find an isomorphism $\delta: H^{1}\left(G, C^{\infty}(\mathbf{H})^{\times}\right) \rightarrow H^{2}(G, \mathbf{Z})$ which corresponds to the Chern class isomorphism by (18) and (19).

The element of $H^{1}\left(G, C^{\infty}(\mathbf{H})^{\times}\right)$corresponding to $\Omega^{1,0}(X)$ is represented by the 1-cocycle $b$, where

$$
b(g)(z)=g^{\prime}\left(g^{-1}(z)\right)
$$

Using (16) and the general theory, we find, for $g, h \in G$,

$$
(\delta b)(g, h)=(1 / 2 \pi i)\left(\log h^{\prime}\left(h^{-1} g^{-1}(z)+\log g^{\prime}\left(g^{-1}(z)\right)-\log (g h)^{\prime}\left((g h)^{-1}(z)\right)\right),\right.
$$

where $z$ is a fixed point in $\mathbf{H}$ and we take the same branch of $\log$ as before. Replacing $z$ by $g h(z)$ and comparing with (4), shows that $\delta b=-a$. Thus the Chern class of $\Omega^{0,1}(X)$ is represented in $H^{2}(G, \mathbf{Z})$ by $\alpha$.

Combining the two calculations of the Chern class and using (18), we see that the proposition is now proved.

We now would like to extend this to an arbitrary Fuchsian group. Suppose that $G$ has the 
presentation (8), (9). Let $L$ be the least common multiple of $e(1), \ldots, e(t)$. Let $C / D$ be the expression of $\tau(G)$ (defined in (11)) as a fraction in lowest terms.

If $G$ has no parabolic elements, by the corollary to Proposition $1, H^{2}(G, \mathbf{Z})=\mathbf{Z} \oplus T(G)$. Let $p_{!}: H^{2}(G, \mathbf{Z}) \rightarrow \mathbf{Z}$ be the projection onto the first factor.

Proposition 3. Notations being as above,

if $s \neq 0$ then $L \cdot \operatorname{Res}{ }_{G}^{\mathfrak{G}}(\alpha)=0$,

but if $s=0$ then $p\left(\operatorname{Res}_{G}^{(\mathfrak{j}}(\alpha)\right) \equiv 0 \quad(\bmod C)$

$$
p\left(\operatorname{Res}{ }_{G}^{(\mathfrak{G}}(\alpha)\right) \neq 0 \text {. }
$$

Proof. If $s \neq 0$, by the corollary to Proposition $1, H^{2}(G, \mathbf{Z})$ is of exponent $L$ and the first statement follows at once.

Suppose that $s=0$. Then by a lemma of Selberg [21, p. 154] there is a normal subgroup $N$ of finite index in $G$ with no elements of finite order. Suppose that the genus of $N$ is $g_{1}$. Then, by (12),

$$
[G: N]=\left(2 g_{1}-2\right) / \tau(G) .
$$

We recall the transfer map (see $[11$, p. 54])

$$
\operatorname{tr}: H^{2}(N, \mathbf{Z}) \rightarrow H^{2}(G, \mathbf{Z}) \text {. }
$$

The characteristic property we require is $([11, \mathrm{p} .56])$ if res is the restriction map from $G$ to $N$ then the composite of

$$
H^{2}(G, \mathbf{Z}) \stackrel{\text { res }}{\rightarrow} H^{2}(N, \mathbf{Z}) \stackrel{\text { tr }}{\rightarrow} H^{2}(G, \mathbf{Z})
$$

is multiplication by $[G: N]$. By the corollary to Proposition $1, H^{2}(N, \mathbf{Z}) \cong \mathbf{Z}$ and

$$
H^{2}(G, \mathbf{Z}) \cong \mathbf{Z} \oplus T(G) \text {. }
$$

Clearly $T(G)$ is of exponent $L$.

Let $\alpha(G)$ (resp. $\alpha(N))$ be $\operatorname{Res}{ }_{G}^{\mathfrak{G}}(\alpha)$ (resp. Res ${ }_{N}^{\mathfrak{G}}(\alpha)$ ). Then res $(\alpha(G))=\alpha(N)$. By Proposition 2 , there is a generator $u$ of $H^{2}(N, \mathbf{Z})$ so that

$$
\alpha(N)=\left(2 g_{1}-2\right) \cdot u \text {. }
$$

But

So

$$
\begin{aligned}
\operatorname{tr}(\operatorname{res}(\alpha(G))) & =[G: N] \alpha(G) \\
& =\left(\left(2 g_{1}-2\right) / \tau(G)\right) \alpha(G)
\end{aligned}
$$

$$
\operatorname{tr}\left(\left(2 g_{1}-2\right) u\right)=\left(\left(2 g_{1}-2\right) / \tau(G)\right) \alpha(G)
$$


If $p(\operatorname{tr}(u))=0$, the composite of maps in (20) would be zero, which is impossible. So $v=p(\operatorname{tr}(u)) \neq 0$. By $(21)$,

Writing $\tau(G)=C / D$ with $(C, D)=1$

$$
v=p(\alpha) G)) / \tau(G)
$$

$$
C \cdot v=D p(\alpha(G))
$$

This proves the proposition.

We now have quite a clear idea of how $\operatorname{Res}{ }_{G}^{\mathbb{G}}(\alpha)$ sits in $H^{2}(G, \mathbf{Z})$, except in respect to the torsion part. We shall now sketch a description of what happens in the torsion part.

Let $G_{i}$ be the cyclic subgroup of $G$ generated by $E_{i}$. Then $H^{2}\left(G_{i}, \mathrm{Z}\right) \cong G_{i}$ (see [11, pp. 40-1]). Thus the restriction morphism from $H^{2}(G, \mathbf{Z})$ to $H^{2}\left(G_{i}, \mathrm{Z}\right)$ gives a homomorphism from $H^{2}(G, \mathbf{Z})$ into $G_{i}$. Combining these we obtain a map

$$
r: H^{2}(G, \mathrm{Z}) \rightarrow \oplus_{j=1}^{t} G_{i}
$$

The right-hand side here is isomorphic to $T(G)$.

If we examine the method of Section 3 closely and follow the proofs in [10], we find that $r$ is the projection of $H^{2}(G, \mathbf{Z})$ onto the factor $T(G)$ given in the corollary to Proposition 1.

We can assume, without loss of generality, that $G_{i}$ is a subgroup of $K$. By (6), we find an explicit form for the restriction of $a$ to $G_{i}$. The resulting element is a generator of $H^{2}\left(G_{i}, \mathrm{Z}\right)$. Thus $r\left(\operatorname{Res}{ }_{G}^{\mathfrak{J}}(\alpha)\right)$ is a generator of each factor of $\underset{1 \leqq j \leqq t}{\oplus} G_{i}$.

5. Liftings. In this section we shall consider the problem of whether $G$ can be lifted to a subgroup of $\mathfrak{G}_{2}=S L(2 ; \mathbf{R})$. The following theorem was originally proved by Petersson ([17]) but was later posed as a problem by Siegel [22]. Bers solved Siegel's problem in [1] in a special case by the theory of Teichmüller spaces. Both Bers and Siegel seem to have been unaware of Petersson's earlier work. The same problem was recently reconsidered in [3].

THEOREM 1. G can be lifted to a subgroup of $S L(2 ; \mathbf{R})$ if and only if it has no elements of order 2.

Proof. The only element of order 2 in $S L(2 ; \mathbf{R})$ is $-I$. So if $G$ had an element of order 2 , it would lift to $-I$. But this projects to the identity of $\mathfrak{G}$. This is a contradiction and it shows that $G$ cannot be lifted.

Now suppose that $G$, with the presentation (8), (9), has no element of order 2 . Then all the $e(j)$ are odd. Thus $L$, the least common multiple of $e(1), \ldots, e(t)$, is odd.

Let us consider first the case when $G$ has parabolic elements; that is when $s>0$. In this case, in $H^{2}(G, \mathbf{Z})$,

$$
L \cdot \operatorname{Res}{ }_{G}^{\mathfrak{G}}(\alpha)=0
$$


Let $a$ be the 2-cocycle defined in (4). By (22) there is a function $b: G \rightarrow \mathbf{Z}$ so that, for $g, h \in G$,

As $L$ is odd, we find:

$$
L \cdot a(g, h)=b(g h)-b(g)-b(h)
$$

$$
a(g, h) \equiv b(g h)-b(g)-b(h)(\bmod 2) .
$$

Thus the image under restriction in $H^{2}\left(G, C_{2}\right)$ of $\alpha$ is zero.

In Section 2 we indicated that $\alpha(\bmod 2)$ induces the double cover $\mathfrak{G}_{2}$ of $\mathfrak{G} . \quad G$ is a subgroup of $\left(\mathfrak{5}\right.$ and so we obtain an extension $G_{2}$ of $G$ which is a subgroup of $\mathfrak{G}_{2}$. But, by (24), the extension is trivial and so $G_{2} \cong G \times C_{2}$. Hence $G$ can be realized as a sugbroup of $\sigma_{2}$. This is the assertion of the theorem.

Now let us consider the case in which $G$ has no parabolic elements; that is, when $s=0$. Let $a$ be the same cocycle as above. As $H^{2}(G, \mathbf{Z}) \cong \mathbf{Z} \oplus T(G)$, we can write an element of $H^{2}(G, Z)$ as $(x, y)$ with $x \in \mathbf{Z}$ and $y \in T(G)$. Suppose that $a$ represents the element $(q, u)$. By Proposition $3, q=C \cdot q_{0}$, where $q_{0} \in \mathbf{Z}$ and $\tau(G)=C / D$ in lowest terms. As

$$
\tau(G)=2 g-2+\sum_{j=1}^{t}\left(1-e(j)^{-1}\right)
$$

and as all the $e(j)$ are odd, $C$ is even. Let $C=2 C_{0}$.

On the other hand $T(G)$ is of exponent $L$ and so $L u=0$. Thus $L \cdot a$ represents $2\left(L \cdot C_{0} \cdot q_{0}, 0\right)$. Let $a^{\prime}$ be a 2 -cocycle representing $\left(L C_{0} q_{0}, 0\right)$. Then there is a function $b: G \rightarrow \mathbf{Z}$ so that

$$
L \cdot a(g, h)=2 \cdot a^{\prime}(g, h)+b(g h)-b(g)-b(h) .
$$

As $L$ is odd, for $g, h \in G$,

$$
a(g, h) \equiv b(g h)-b(g)-b(h)(\bmod 2) .
$$

This is the same congruence as (24) and the deduction of the theorem is just as before. Thus the theorem is proved.

We have restricted our attention to one particular extension, that of $\mathfrak{G}$ to $\mathfrak{G}_{2}$. Clearly the method is more generally applicable but the results are neither of such interest nor of such simplicity.

6. The theory of weights. In this section we shall sketch a version of Petersson's theory of weights and multipliers for an arbitrary Fuchsian group. It should be pointed out at the start that Petersson works with discrete subgroups of $S L(2 ; \mathbf{R})$ rather than of $P S L(2 ; \mathbf{R})$. Consequently his theory is not directly comparable. A discussion of Petersson's theory from a point of view closer to our own is given by Roelcke in [18].

Let $\alpha(n)$ be the class of $a(\bmod n)$ in $H^{2}\left(\mathfrak{5}, C_{n}\right)$. We allow $n$ to be $\infty$ in which case $\alpha$ is the class of $a$ in $H^{2}\left((5, Z)\right.$. In future if $n=\infty$ we understand $C_{n}$ to be Z. $\alpha(n)$ determines the extension

$$
e \rightarrow C_{n} \rightarrow \mathfrak{W}_{n} \rightarrow \mathfrak{W} \rightarrow e
$$


Let $N$ be an integer. Then let $\mathscr{G}_{n}(N)$ be the group defined by the extension

$$
e \rightarrow C_{n} \rightarrow \mathfrak{W}_{n}(N) \rightarrow \mathfrak{W} \rightarrow e
$$

determined by $N \alpha(n) . \quad \mathfrak{G}_{n}(N)$ is not necessarily connected. In fact it has $(n, N)(N$ if $n=\infty)$ connected components. The component of the identity is isomorphic to $\mathfrak{b}_{d}$, where $d=n /(n, N)(d=\infty$ if $n=\infty$ and $N \neq 0)$. These facts may easily be verified by the methods of Section 2 by means of checking them first for $K$. The structure of $\mathfrak{G}_{n}(N)$ depends only on $n$ and $(n, N)$. Let $\delta \mid n$ and set

$$
\boldsymbol{G}_{n, \delta}=\mathfrak{G}_{n}(\mathfrak{n} / \delta)
$$

Then $\mathfrak{G}_{n, \delta}$ has $n / \delta$ components and the identity component is isomorphic to $\mathfrak{b}_{\delta}$. In fact we can describe $\mathfrak{G}_{n, \delta}$ completely. The centre of $\mathfrak{G}_{\delta}$ is isomorphic to $C_{\delta}$. Let $\gamma$ be a generator of this group. We construct the group generated by $\mathfrak{5}_{\delta}$ and another symbol $\varepsilon$ which commutes with every element of $\mathfrak{5}_{\delta}$ and is such that

$$
\varepsilon^{n / \delta}=\gamma
$$

The resulting group is isomorphic to $\mathfrak{F}_{n, \delta}$.

Let $G$ be a Fuchsian group. We say that $\delta$ is a divisor of $G$ if there is a non-zero integer $n$ such that

$$
n \cdot \operatorname{Res}{ }_{G}^{\sigma}(\alpha(n \delta))=0
$$

From the discussion above and the general theory we have:

Proposition 4. If $\delta$ is a positive integer or $\infty$ then $\delta$ is a divisor of $G$ if and only if $G$ can be lifted to some group of the form $\boldsymbol{6}_{n, \delta}$.

The central proposition which both justifies the notation and shows the connection with Petersson's theory is the following:

Proposition 5. Let $S=\mathbf{R} / \mathbf{Z}$ and let $\delta$ be a non-zero integer. Let $(\alpha / \delta)$ be the class of $\delta^{-1} a$ in $H^{2}(G, S)$. Then $\delta$ is a divisor of $G$ if and only if $(\alpha / \delta)=0$.

Proof. Suppose that $\delta$ is a divisor of $G$. Then there is an integer $n$ and a function $b: G^{\prime} \rightarrow \mathbf{Z}$ so that, for $g, h \in G$,

Thus

$$
n \cdot a(g, h) \equiv b(g h)-b(g)-b(h)(\bmod n \delta) .
$$

$$
a(g, h) / \delta \equiv(b(g h) / n \delta)-(b(g) / n \delta)-(b(h) / n \delta)(\bmod 1) .
$$

So $(\alpha / \delta)=0$ in $H^{2}(G, S)$.

Conversely suppose that there is $B: G \rightarrow \mathbf{R}$ so that

$$
a(g, h) / \delta \equiv B(g h)-B(g)-B(h)(\bmod 1) .
$$

Let $V$ be the subgroup of $\mathbf{R}$ generated by $\{B(g): g \in G\}$ and $\delta^{-1}$. Let $g_{1}, g_{2}, \ldots, g_{K}$ be a set of generators of $G$. By the congruence above, $V$ is generated by $B\left(g_{1}\right), \ldots, B\left(g_{K}\right)$ and $\delta^{-1}$. 
Thus $V$ is a finitely generated torsion-free abelian group. From the general theory of abelian groups it follows that there is a projection of $V$ onto $V \cap \mathbf{Q}$. Call this projection $p$ and suppose that $V \cap \mathbf{Q}$ generated by $J^{-1}$ (it must be generated by such an element as $1 \in V$ ). Then

$$
a(g, h) / \delta \equiv p(B(g, h))-p(B(g))-p(B(h))(\bmod 1) .
$$

If we set $b(g)=J \delta p(B(g))$, then $b(g)$ is an integer and

$$
J \cdot a(g, h) \equiv b(g h)-b(g)-b(h)(\bmod J \delta) .
$$

Thus $\delta$ is a divisor of $G$ and the proposition is proved.

COROLlaRY If $d$ is a divisor of $G$ and $d_{1} \mid d$ then $d_{1}$ is a divisor of $G$. If $d_{1}, d_{2}$ are divisors of $G$ and $\left(d_{1}, d_{2}\right)=1$ then $d_{1} d_{2}$ is a divisor of $G$.

Proof. This is obvious from Proposition 5.

This corollary justifies the use of the word divisor. The following examples are consequences of the results of Section 3. As usual, $G$ has the presentation (8), (9) and $L$ is the least common multiple of $e(1), \ldots, e(t)$.

EXAMPLES. (i) If $s=t=0$, the divisors of $G$ are the divisors of $2 g-2$.

(ii) If $s \neq 0$, then every integer and $\infty$ is a divisor of $G$.

(iii) If $s=0$, then any divisor of $C$ (defined above) is a divisor of $G$.

In this connection it is worth noting that, by Proposition $1, H^{2}(G, S) \cong S$ if $s=0$ and $H^{2}(G, S)=0$ if $s \neq 0$.

Suppose that $G$ can be lifted to $\mathfrak{G}_{n, \delta}$. Then it is clearly of interest to study functions on $G \backslash \mathfrak{G}_{n, \delta}$ in the style of [6], [19] and the interpretation of such functions as automorphic forms should also be useful ([18]). This is too large a topic for us to embark on here.

We shall now review Petersson's theory. If $u \neq 0$ and $v$ are complex numbers, we shall write $u^{v}$ for $\exp (v \log u)$, where we use the same branch of $\log$ as before. Let $V$ be a complex vector space and let $m: G \rightarrow \operatorname{End}(V)$ be a function. We say that $f: \mathbf{H} \rightarrow V$ is an automorphic form of weight $k(\in \mathbf{R})$ and multiplier $m$ if

For $g, h \in \mathfrak{G}$, let

$$
f(g(z)) \cdot g^{\prime}(z)^{k}=m(g) f(z)
$$

$$
q(g, h)=\exp (2 \pi i k a(g, h)) \text {. }
$$

Then a necessary and sufficient condition for (27) to be consistent is that, for $g, h \in G$,

$$
q(g, h) \cdot I d_{V}=m(g h) m(h)^{-1} m(g)^{-1} .
$$

A full study of (29) seems difficult but interesting. It is connected with the theory of vector bundles on a Riemann surface and the reader may be referred to [24], [15]. We shall consider only the case when $V$ is 1-dimensional and $|m(\cdot)|=1$, in which case $m$ is said to be unitary.

$q$ is a 2-cocycle with values in the circle group $S$. Suppose in the presentation (8), (9) that 
$s \neq 0$. As $H^{2}(G, S)$ is trivial, (29) can be solved for any real weight $k$. On the other hand, if $s=0$, then $k$ must be a multiple of $\delta^{-1}$ where $\delta$ is a divisor of $G$ and thus take on a finite set of values $(\bmod 1)$. In the special case $s=t=0$ all weights are multiples of $(2 g-2)^{-1}$. In any case there is an integer $N$ such that every weight is a multiple of $N^{-1}$.

In the next section we shall construct forms of various weights which show more directly how the phenomena noted in this section occur.

7. Construction of functions. Let $G$ be a Fuchsian group of the first kind acting on $\mathbf{H}$. We are going to construct automorphic forms, in Petersson's sense. The method we shall follow is much more intrinsic than the use of Poincare series.

Let $D=y^{2}\left(\partial^{2} / \partial x^{2}+\partial^{2} / \partial y^{2}\right)$ be the Laplace-Beltrami operator on $\mathbf{H}$. For $\operatorname{Re}(s)>1$, let $G\left(z_{1}, z_{2}, s\right)$ be the resolvent kernel for $D$ on $G \mid \mathbf{H}$. This is constructed in [4]. $G\left(z_{1}, z_{2}, s\right)$ satisfies

(i) if $z_{1} \neq z_{2}$ then $D_{z_{1}} G\left(z_{1}, z_{2}, s\right)=-s(1-s) G\left(z_{1}, z_{2}, s\right)$,

(ii) $G\left(z_{1}, z_{2}, s\right)=G\left(z_{2}, z_{1}, s\right)$,

(iii) if $g \in G, G\left(g\left(z_{1}\right), z_{2}, s\right)=G\left(z_{1}, z_{2}, s\right)$,

(iv) if the order of the subgroup of $G$ fixing $z_{2}$ is $e\left(z_{2}\right)$, then

$$
G\left(z_{1}, z_{2}, s\right)+\left(e\left(z_{2}\right) / 2 \pi\right) \log \left|z_{1}-z_{2}\right|
$$

is real-analytic as a function of $z_{1}$ near $z_{2}$.

Suppose that $G$ has no parabolic elements. Let $\varphi_{0}, \varphi_{1}, \ldots$ be a complete orthonormal system of eigenfunctions of $D$ invariant under $G$. Let

$$
D \varphi_{j}=-\mu_{j} \varphi_{j}
$$

Suppose that the $\mu_{j}$ are so ordered that $\mu_{0} \leqq \mu_{1} \leqq \mu_{2} \leqq \ldots$. Then it is known (see [13]) that $\mu_{0}=0$ and that $\mu_{1}>0$. Furthermore $\varphi_{0}$ is a constant and so

$$
\begin{aligned}
\varphi_{0} & =\sigma(G \mid \mathbf{H})^{-1 / 2} \\
& =(2 \pi \tau(G))^{-1 / 2} .
\end{aligned}
$$

It also follows from the general theory that $\sum_{j \neq 0} \mu_{j}^{-2}<\infty$. Let $s_{0}$ be an arbitrary complex number with $\operatorname{Re}\left(s_{0}\right)>1$. Then for any $s$ we have the eigenfunction expansion, on setting $\mu=s(1-s)$ and $\mu^{\prime}=s_{0}\left(1-s_{0}\right)$,

$$
G\left(z_{1}, z_{2}, s\right)-G\left(z_{1}, z_{2}, s_{0}\right)=\sum_{j=0}^{\infty}\left(\mu-\mu^{\prime}\right) \varphi_{j}\left(z_{1}\right) \varphi_{j}\left(z_{2}\right) /\left(\mu_{j}-\mu\right)\left(\mu_{j}-\mu^{\prime}\right) .
$$

The right-hand side converges to an $L^{2}$ function analytic in $s$ except when $s(1-s) \in\left\{\mu_{j}\right\}$. Also, at $s=1$ there is a pole of residue $\varphi_{0}^{2}=1 / 2 \pi \tau(G)$.

If $G$ has parabolic elements, it follows from the decomposition of $L^{2}(G \backslash \mathbf{H})$ by Eisenstein series (see [5], [18] and [20]) that $G\left(z_{1}, z_{2}, s\right)-G\left(z_{1}, z_{2}, s_{0}\right)$ is analytic in $s$ as an $L^{2}$ function, 
except for a possible finite set of simple poles on $\operatorname{Im}(s)=0$. Furthermore, as the continuous spectrum only contributes if $\operatorname{Re}(s)=1 / 2$, the pole at $s=1$ is as before.

Suppose that $G\left(z_{1}, z_{2}, s\right)$ has the following expansion

$$
G\left(z_{1}, z_{2}, s\right)=(2 \pi \tau(G)(s-1))^{-1}+a_{0}\left(z_{1}, z_{2}\right)+a_{1}\left(z_{1}, z_{2}\right)(s-1)+\ldots
$$

Let $C$ be a small circle around 1 so that $C$ lies in $\operatorname{Re}(s)>1 / 2$ and there is no pole of $G\left(z_{1}, z_{2}, s\right)$ other than $s=1$ inside $C$. Let $\varepsilon_{j}=1$ if $j=0$ and $\varepsilon_{j}=0$ if $j>0$. Then, for $j \geqq 0$,

$$
a_{j}\left(z_{1}, z_{2}\right)-\varepsilon_{j} G\left(z_{1}, z_{2}, s_{0}\right)=(1 / 2 \pi i) \int_{C}\left(G\left(z_{1}, z_{2}, s\right)-G\left(z_{1}, z_{2}, s_{0}\right)(s-1)^{-j-1} d s .\right.
$$

By conditions (i), (ii) and (iv), the integrand is a real-analytic function of $z_{1}$ and $z_{2}$. Thus if $j>0, a_{j}\left(z_{1}, z_{2}\right)$ is a real-analytic function of $z_{1}, z_{2}$, whereas

is real-analytic in $z_{1}$ near $z_{2}$.

$$
a_{0}\left(z_{1}, z_{2}\right)+\left(e\left(z_{2}\right) / 2 \pi\right) \log \left|z_{1}-z_{2}\right|
$$

The integral above could have been used as a definition of $a_{j}\left(z_{1}, z_{2}\right)$. From property (i) above it follows at once that

We have now shown

$$
D_{z_{1}} a_{0}\left(z_{1}, z_{2}\right)=1 / 2 \pi \tau(G)
$$

Proposition 6. There exists a function a on $\mathbf{H} \times \mathbf{H}$ with the following properties:

(i) If $g \in G$, then $a\left(g\left(z_{1}\right), z_{2}\right)=a\left(z_{1}, z_{2}\right)$.

(ii) $a\left(z_{1}, z_{2}\right)=a\left(z_{2}, z_{1}\right)$.

(iii) If $z_{1} \notin G\left\{z_{2}\right\}$, then $a\left(w, z_{2}\right)$ is real analytic in $w$ in a neighbourhood of $z_{1}$. Otherwise

$$
a\left(w, z_{2}\right)+e\left(z_{2}\right) \cdot \log \left|w-z_{2}\right|
$$

is real analytic with $w$ in a neighbourhood of $z_{2}$.

(iv) $D_{w} a\left(w, z_{2}\right)=\tau(G)^{-1}$.

(v) As $s \rightarrow 1$,

$$
G\left(z_{1}, z_{2}, s\right)=(2 \pi)^{-1}\left(\tau(G)^{-1}(s-1)^{-1}+a\left(z_{1}, z_{2}\right)+0(s-1)\right) .
$$

Suppose now that $G$ has parabolic elements. Let $p$ be a parabolic fixed point of $G$ and let $E_{p}(z, s)$ be the Eisenstein series associated to $p([20])$. This has an analytic continuation to $\operatorname{Re}(s)>1 / 2$ except for a finite set of poles on the real axis ([18], [20]). There is a pole of multiplicity 1 at $s=1$. The residue at such a pole is a square-integrable eigenfunction of $D$; if the pole is at $s=s_{1}$ then the eigenvalue is $-s_{1}\left(1-s_{1}\right)$. In particular the residue at $s=1$ is a constant. As before, we may expand $E_{p}(z, s)$ as

$$
E_{p}(z, s)=c /(s-1)+a_{0}(z)+a_{1}(z)(s-1)+\ldots
$$

and again we find that $a_{0}$ is real analytic and $D a_{0}=c$. The value of $c$ may be calculated from the Maaß-Selberg relations (see [8], [20]). The result is that

$$
c=1 /(2 \pi \tau(G)) \text {. }
$$


Define now the Poisson " kernel " for $\mathbf{H}$ as follows: if $z \in \mathbf{H}, x \in \mathbf{R}$, set

and

$$
P(z, x)=\operatorname{Im}(z) /|z-x|^{2}
$$

$$
P(z, \infty)=\operatorname{Im}(z) \text {. }
$$

Then from the Fourier expansion of $E_{p}(z, s)$ we obtain, as $z \rightarrow p$ through a cusp

$$
a_{0}(z)=P(z, p)+0(\log |P(z, p)|) .
$$

On the other hand if $q$ is a parabolic fixed point of $G$ which is equivalent to $p$, then, as $z \rightarrow q$ through a cusp,

$$
a_{0}(z)=0(\log |P(z, q)|) .
$$

$a_{0}$ is a limiting version of $a\left(z_{1}, z_{2}\right)$ as $z_{2} \rightarrow p$. This can easily be proved rigorously but we shall here concentrate on $a\left(z_{1}, z_{2}\right)$ and only sketch in the corresponding theory for $a_{0}$. This idea is also mentioned briefly in [23, \$26.3].

To cast the results above into a more recognizable form, we make use of the following lemma:

LEMMA. Let $F$ be a real function on $\mathbf{H}$ satisfying

(i) for $g \in G F(g(z))=F(z)$,

(ii) for some constant $c, D F=c$,

(iii) there is a point $w \in \mathbf{H}$ and an integer e such that if $z$ is not equivalent under $G$ to $w$, then $F$ is real analytic in a neighbourhood of $z$; whereas

$$
F(u)+e \log |u-w|
$$

is real analytic in a neighbourhood of $w$.

Then there is a function $m$ on $G$ such that $|m|=1$, and an analytic function $\varphi$ such that, for $g \in G$,

$$
\varphi(g(z)) \cdot g^{\prime}(z)^{c}=\varphi(z) \cdot m(g)
$$

and $\varphi$ has zeros of order $e$ at points equivalent to $w$ and no others. Also

$$
-F(z)=\log |\varphi(z)|+c \cdot \log \operatorname{Im}(z) .
$$

Proof. Let $F_{1}(z)=F(z)+c \cdot \log \operatorname{Im}(z)$. Then one checks that $D F_{1}=0$; thus $F_{1}$ is harmonic. Thus locally there is an analytic function $\varphi_{1}$ such that

$$
F_{1}(z)=\operatorname{Re}\left(\varphi_{1}(z)\right) .
$$

On going around $w$ (or a point equivalent to $w$ ), $\varphi_{1}$ changes by an integral multiple of $2 \pi i$. Thus if we set

$$
\varphi(z)=\exp \left(-\varphi_{1}(z)\right)
$$

it can be analytically continued to $\mathrm{H}$ and it is standard to check that the resulting function has all the properties ascribed to it in the lemma. 
COROLlaRY. The function $m$ appearing in the lemma is a multiplier of weight $c$ (in the sense of Petersson).

Proof. This follows from the lemma and the discussion at the end of Section 6.

THEOREM 2. Let $w$ be a point of $\mathbf{H}$. Then there is an analytic automorphic form $\varphi(z ; w)$ of weight $(e(w) \tau(G))^{-1}$ with a simple zero at points equivalent to $w$ and no other zeros or poles. Let $m_{w}$ be the multiplier for $\varphi(z ; w)$. Then $m_{z}^{e(z)}$ depends real-analytically on $z$. If $g \in G$, then $\varphi(z ; g(w))$ is a multiple of $\varphi(z ; w)$.

Proof. This follows from the lemma and Proposition 6.

The same construction applied to $a_{0}$ shows that there is an analytic automorphic form $\eta_{p}$ of weight $\tau(G)^{-1}$. $\eta_{p}$ has no zeros or poles. From (31) and (32) we deduce that the " $q$-series" at $p$ is of the form $q+0\left(q^{2}\right)$ but at a parabolic vertex inequivalent to $p$ the series is of the form $1+0(q)$. This is the function constructed by Goldstein [8].

This shows that we have both generalized and have given a new proof of the Kronecker limit formula.

The theorem is our basic existence theorem. It shows that if $r_{1}, \ldots, r_{t}$ are integers, then there is a form of weight

$$
\sum_{j=1}^{t}\left(r_{j} / e(j)\right) \tau(G)^{-1} .
$$

That is to say, there is a form of every weight which is a multiple of $(L \tau(G))^{-1}$. Observe that $L \tau(G)$ is an integer.

The existence of $\eta_{p}$ shows that if $G$ has parabolic elements, then, if we ignore the behaviour at cusps, all real weights can occur.

If $s=t=0$, then $L \tau(G)=2 g-2$, which shows that we have established the existence of forms of all possible weights.

If we ignored the behaviour of the fixed points, then we would only obtain forms whose weights were multiples of $\tau(G)^{-1}$. In this way we see darkly a relation between the fixed points of $G$ and the cohomology of $G$.

Using Theorem 2, it is possible for us to complete our earlier investigations. For the rest of this section we shall assume that $s=0$.

COROLlaRY 1. Suppose that $s=0$ and that $f$ is a meromorphic form of weight $k$ with a unitary multiplier. Then there are points $w_{1}, \ldots, w_{N} \in \mathbf{H}$, integers $r(1), \ldots, r(N)$ and a constant c such that

$$
f(z)=c \cdot \prod_{j=1}^{N} \varphi\left(z, w_{j}\right)^{r(j)} .
$$

Proof. Let $w_{1}, \ldots, w_{N}$ be the zeros of $f$ in some fundamental domain for $G$ and let their multiplicities be $r(1), \ldots r(N)$ respectively, poles being counted as zeros of negative multiplicity. Set

$$
f_{1}(z)=\prod_{j=1}^{N} \varphi\left(z, w_{j}\right)^{r(j)}
$$


Then $f / f_{1}$ is a form of some weight $k_{1}$. If we count the contributions from elliptic elements with their usual fractional values, we have, by an easy extension of lemma VI.4.3 of [9],

$$
\operatorname{deg}\left(\left(f / f_{1}\right)\right)=k_{1} \tau(G)
$$

As $f / f_{1}$ has no zeros or poles it follows that $k_{1}=0$. Thus, in the usual language, $f / f_{1}$ is a multiplicative function. As $f / f_{1}$ has no poles or zeros its character is inessential. But by construction it is also unitary. Hence it is trivial. Thus $f / f_{1}$ must be a constant. This proves the corollary.

CoROLLARY 2. Every weight is of the form $n(L \tau(G))^{-1}(n \in \mathbf{Z})$.

Proof. Let $m$ be a multiplier of weight $k$. Then, as is well known (see $[4, \$ 10])$, we can construct a meromorphic form $f$ of weight $k$ with multiplier $m$ as a quotient of two Poincaré series. The conclusion follows from Corollary 1 and the remarks after Theorem 2.

In particular this shows that the best possible form of Proposition 3 is with $C$ replaced by $L \tau(G)$. Also it shows that $\delta$ is a divisor of $G$ if and only if $\delta$ is a divisor of $L \tau(G)$. This corollary was first proved by Petersson [17].

8. Applications to Riemann surfaces. We show in this section how Theorem 2 can be applied to the function theory on a compact Riemann surface $X$ of genus $g>1$. Let $G$ be a Fuchsian group uniformizing $X$ so that

$$
X \cong G \mid \mathbf{H} .
$$

Then Theorem 2 shows that, for $P \in X$, there is a form $\varphi_{P}$ of weight $(2 g-2)^{-1}$ with multiplier $m \mathfrak{a}$ and a simple zero at $P$. Let $a$ be a divisor on $X$. Then if $a=\sum n_{j} P_{j}$, set

and

$$
\varphi_{\mathfrak{a}}=\prod\left(\phi_{P_{j}}\right)^{n_{j}}
$$

$$
m_{\mathfrak{a}}=\prod\left(m_{P_{j}}\right)^{n_{j}} .
$$

Then $\varphi_{\mathfrak{a}}$ is a form of weight $\operatorname{deg}(\mathfrak{a}) /(2 g-2)$ and multiplier $m_{\mathfrak{a}}$. Also

$$
\left(\varphi_{\mathfrak{a}}\right)=\mathfrak{a} .
$$

Let $L(a)$ be the space of holomorphic functions, so that

$$
(f) \leqq-a \text {. }
$$

Then $f \in L(\mathfrak{a})$ if and only if $f \varphi_{\mathfrak{a}}$ is a holomorphic form with multiplier $m_{\mathfrak{a}}$ and of weight $\operatorname{deg}(\mathfrak{a}) /(2 g-2)$.

Let $S(m, k)$ be the space of holomorphic forms of weight $k$ and multiplier $m$. Then we have shown

$$
S\left(m_{\mathfrak{a}}, \operatorname{deg}(\mathfrak{a}) /(2 g-2)\right) \cong L(\mathfrak{a}) .
$$

Then, for instance, the Riemann-Roch Theorem becomes

$$
\operatorname{dim}(S(m, k))-\operatorname{dim}(S(\bar{m}, 1-k))=(2 k-1)(g-1) .
$$


By analytic means (such as the Selberg trace formula or index theorems) it is easy to prove the Riemann-Roch Theorem in this form.

If $a$ is of degree 0 , then $m_{\mathfrak{a}}$ is a character on $G$. The group of all characters on $G$ may be identified with the Jacobian $J(X)$ of $X$. So we have an explicit map from the divisors of degree 0 to $J(X)$. On comparing we find that this is the usual Abel map (see [9]).

The importance of this is that this gives a tentative connection between the spectral decomposition of the Laplace-Beltrami operator and the classical theory of functions. But we shall not pursue this topic here.

9. The modular group. In the case of the modular group we can make the forms $\varphi$ and $\eta_{p}$ more explicit. We shall consider $\eta_{\infty}$ first. At the end of Section 7 we showed that $\eta_{\infty}$ is a cusp form of weight $\tau(G)^{-1}=6$. Thus $\eta_{\infty} / \Delta$, where $\Delta$ is the usual discriminant form, is an automorphic function with some character $\beta$ and having no poles or zeros. The modular group is generated by an element of order 3 and an element of order 2 . Thus any character is of order 6 . So $\left(\eta_{\infty} / \Delta\right)^{6}$ is a function with trivial multiplier and no poles or zeros. Hence $\left(\eta_{\infty} / \Delta\right)^{6}$ is a constant. Thus $\eta_{\infty}$ is a multiple of $\Delta$. This is the essential content of the Kronecker limit formula. There is only a matter of constants to settle. The importance of this method of proof is that it relies very little on the special features of the problem and hence should admit wide extension.

In the same way one can show that, up to a constant, multiple,

$$
\varphi(z ; w)=((j(z)-j(w)) \Delta(z))^{1 / e(w)} .
$$

The multiplier is the $(24 / e(w))$ th power of the multiplier of the Dedekind $\eta$-function.

\section{REFERENCES}

1. L. Bers, Spaces of Riemann surfaces, Proc. Int. Cong. Math. Edinburgh (Cambridge, 1960).

2. S. S. Chern, Complex manifolds without potential theory (Van Nostrand, 1967).

3. J. Dyer and J. Lewittes, Möbius transformations and matrices (Preprint).

4. J. Elstrodt, Die Resolvente zum Eigenwertproblem der automorphen!Formen in der hyperbolischen Ebene, I, II, III, Math. Ann. 203 (1973), 295-330, Math. Zeit. 132 (1973), 99-134, Math. Ann. 208 (1974), 99-132.

5. L. D. Fadeev, Expansion in eigenfunctions of the Laplace operator on the fundamental domain of a discrete group on the Lobačevskii plane. Trans. Moscow Math. Soc. 17 (1967), 357-386.

6. I. M. Gel'fand, M. I. Graev and I. I. Pyatetskii-Shapiro, Representation theory and automorphic functions (Saunders, 1969).

7. R. Godement, Cohomology des groupes discontinues (Sém. Bourbaki (1954) No. 90).

8. L. J. Goldstein, Dedekind sums for a Fuchsian group, I., Nagoya Math. J. 50 (1973), 21-47.

9. I. Kra, Automorphic forms and Kleinian groups (Benjamin, 1972).

10. A. G. Kurosh, The theory of groups, Vol. 2 (Chelsea, 1956).

11. S. Lang, Rapport sur la cohomologie des groupes (Benjamin, 1966).

12. A. Leutbecher, Uber Automorphiefaktoren und die Dedekindschen Summen, Glasgow Math. J. 11 (1970), 41-57.

13. H. P. McKean, Selberg's trace formula as applied to a compact Riemann surface, Comm. Pure Appl. Math. XXV (1972), 225-246. 
14. D. Mumford, Abelian varieties (Oxford, 1970).

15. M. S. Narasimhan and C. S. Seshadri, Stable and unitary vector bundles on a compact Riemann surface, Ann. Math. 82 (1965), 540-567.

67.

16. H. Petersson, Zur analytischen theorie der Grenzkreisgruppen, I., Math. Ann. 115 (1938), 23-

17. H. Petersson, Zur analytischen theorie der Grenzkreisgruppen, III., Math. Ann. 115 (1938), $518-572$.

18. W. Roelcke, Das Eigenwertproblem der automorphen Formen in der hyperbolischen Ebene, I, II, Math. Ann. 167 (1966), 292-337, Math. Ann. 168 (1967), 261-234.

19. A. Selberg, Harmonic analysis and discontinuous groups in weakly symmetric Riemannian spaces with applications to Dirichlet series, J. Indian Math. Soc. 20 (1956), 47-87.

20. A. Selberg, Discontinuous groups and harmonic analysis, Proc. Int. Cong. Math. (Stockholm, 1962), 177-189.

21. A. Selberg, On discontinuous groups in higher dimensional symmetric spaces. Contributions to Functions Theory (Bombay, 1960), 147-164.

22. C. L. Siegel, Ưber einige Ungleichungen bei Bewegungsgruppen in der nichteuklidischen Ebene, Math. Ann. 133 (1957), 127-138.

23. C. L. Siegel, Analytische Zahlentheorie, II. Lecture notes (Wintersemester 1963/4, Göttingen).

24. A. Weil, Généralisation des fonctions abéliennes, J. Math. Pures et Appl. 17 (1938), 47-87.

Department of Pure Mathematics and Mathematical Statistics

16 Mill LANE

CAMBridge, CB2 1SB 